\title{
Is Antimicrobial Susceptibility Testing Necessary Before First-line Treatment for Helicobacter pylori Infection? -Meta-analysis of Randomized Controlled Trials-
}

\author{
Yuan Wenzhen ${ }^{1,2}$, Li Yumin ${ }^{3}$, Guan Quanlin ${ }^{1}$, Yang Kehu ${ }^{2}$, Jiang Lei ${ }^{1}$, \\ Wang Donghai ${ }^{4}$ and Yang Lijuan ${ }^{5}$
}

\begin{abstract}
Background With the wide use of antibiotics, antibiotic-resistant Helicobacter pylori strains are becoming increasingly prevalent. It has been hypothesized that culture-guided therapy might help to increase treatment success. But the effects and the costs still remain controversial.

Aims To systematically review the efficacy and the cost of culture-guided triple therapy, compared to standard triple regimen for first-line treatment of Helicobacter pylori infection.

Methods A search of the Cochrane Library, PubMed, EMBASE, Science Citation Index Expanded and CBM was performed. Randomized controlled trials comparing culture-guided triple therapy to standard triple therapy in the first-line treatment of Helicobacter pylori infection were selected for meta-analysis. Relative risk was used as a measure of the effect of two regimens mentioned above with a fixed-effects model using the methods of DerSimonian and Laird.

Results Five randomized controlled trials totaling 701 patients were included. The meta-analysis showed that culture-guided triple therapy was superior referring to a higher eradication rate from intention-to-treat analyses (RR, 0.84; 95\% CI,0.77, 0.90; $\mathrm{p}<0.00001)$ and a lower overall cost.

Conclusion Culture-guided triple therapy was more effective than standard triple therapy for first-line treatment of Helicobacter pylori infection. Based on the only paper focused on the overall cost, the cultureguided triple therapy was also more cost saving. Antimicrobial susceptibility testing is necessary before firstline treatment for Helicobacter pylori infection.
\end{abstract}

Key words: Helicobacter pylori, first-line treatment, culture-guided triple therapy, standard triple therapy, overall cost, Meta-Analysis

(Inter Med 49: 1103-1109, 2010)

(DOI: 10.2169/internalmedicine.49.3031)

\section{Introduction}

Helicobacter pylori $(H$. pylori) is a highly prevalent chronic infection with a worldwide prevalence of nearly $50 \%$, U.S. prevalence of $30-40 \%(1,2)$, and Chinese prevalence of $45 \%$ (3). H. pylori is known to play a major contributory role in the pathogeneses of chronic gastritis, peptic ulcers, gastric mucosa-associated lymphoid tissue (MALT) lymphoma and distal gastric cancer (4-6).

Although various antimicrobial regimens have been proposed and tested, the regimen combining a proton pump inhibitor or ranitidine bismuth citrate with two antibiotics for 7 to 14 days triple therapies is the recommended first-line treatment for $H$. pylori infection (7-9). This standard therapy presents eradication rates of $H$. pylori from $60 \%$ up to 90\% (10-13), and the major obstacle to $100 \%$ effective therapy being represented by antimicrobial-resistant $H$. pylori

\footnotetext{
${ }^{1}$ The first hospital of Lanzhou University, Lanzhou, China, ${ }^{2}$ The Evidence-Based Medicine Center of Lanzhou University, Lanzhou, China, ${ }^{3}$ The second hospital of Lanzhou University, Key laboratory of digestive system tumors, Gansu province, Lanzhou, China, ${ }^{4}$ Montefiore Medical Center, Albert Einstein College of Medicine, New York, U.S.A. and ${ }^{5}$ The school of Economics of Lanzhou University, Lanzhou, China Received for publication October 17, 2009; Accepted for publication January 28, 2010 Correspondence to Dr. Li Yumin, liyumin168@yahoo.com.cn
} 
strains $(12,14)$. Metronidazole resistance was the first to be described $(15,16)$ and clarithromycin resistance was another key factor (17). In recent reports, resistance ranged from $10 \%$ to $50 \%$ for metronidazole and $0 \%$ to $15 \%$ for clarithromycin in Europe $(18,19)$, whereas, in the USA, $37 \%$ for metronidazole and $10 \%$ for clarithromycin 20 ), and it varies widely amongst different ethnic groups $(20,21)$.

However, with the wide use of such antibiotics, antibioticresistant $H$. pylori strains are becoming increasingly prevalent (14). Several studies have evaluated the relationship between pre-treatment antibiotic resistance and eradication rate using different standard treatments and performing a susceptibility test before the initiation of the therapeutic regimen (22-25). Accumulating evidences [included randomized controlled trials (RCTs)] suggest antibiotic sensitivity testing results in improving eradication rate (26-31). Nevertheless, the effects still remain controversial (32-34). Furthermore, whether or not culture-guided therapy is cost saving is another bone of contention, compared to standard treatment $(30,31,35,36)$.

We systematically reviewed all RCTs and carried out a meta-analysis to assess whether a culture-guided triple therapy (chosen based on a preliminary in vitro susceptibility test), and a standard triple therapy could improve the eradication rate in patients affected by $H$. pylori infection and to assess which regimen is cost saving.

\section{Patients and Methods}

\section{Study selection criteria}

The titles and abstracts of all citations identified by the literature search were reviewed. Selection criteria were then applied to all potentially relevant studies. The selection criteria for inclusion in the meta-analysis were: (i) RCTs (included quasi-randomized controlled trials) comparing two first-line therapies; (ii) They had to include at least two branches of treatment consisting of (a) standard triple therapy that included a proton pump inhibitor or a ranitidine bismuth citrate with two antibiotics for 7 to 14 days, and (b) culture-guided triple therapy that included a proton pump inhibitor or a ranitidine bismuth citrate with two antibiotics chosen based on susceptibility testing for 7 to 14 days; (iii) Confirmation of $H$. pylori eradication at least 4 weeks after completion of treatment; (iv) The confirmation of infection based on urea breath testing.

\section{Search strategy for identification of studies}

Trials were identified by searching the Cochrane Library (Issue 4 2008), PubMed (December 2008), EMBASE (December 2008), Science Citation Index Expanded (December 2008), and CBM (Chinese Biomedical Literature Database) (December 2008). A search strategy was constructed by using a combination of the following words: (Helicobacter pylori OR H. pylori) AND (culture OR susceptibility OR antimicrobial sensitivity OR vitro susceptibility testing OR an- tibiotic). Articles published in any language were included. Reference lists from the trials selected by electronic searching were hand searched to identify further relevant trials. Abstracts of the articles selected in each of these multiple searches were reviewed and those meeting the inclusion criteria were recorded. In the case of duplicate reports, or studies obviously reporting results from the same study population, only the latest published results were used.

\section{Assessment of study quality}

Two investigators (Yuan Wenzhen and Li Yumin) conducted the search independently and they also evaluated study quality using the simple method that is recommended by the Cochrane Handbook (37). Quality assessment of studies was performed independently by two reviewers (Yuan Wenzhen and Li Yumin). Discrepancies in the interpretation were resolved by consensus.

\section{Data extraction}

Two investigators (Yuan Wenzhen and Li Yumin) extracted the data from the studies meeting the selection criteria. Data were extracted concerning (a) study design; (b) age and gender of patients enrolled in the study; (c) number of patients enrolled in the study; (d) testing used to confirm persistent infection prior to study enrollment and the eradication after treatment; (e) drug regimen, including specific doses and treatment duration; (f) number of patients in which $H$. pylori infection was successfully eradicated (either directly provided or calculated given the intention-to-treat and per-protocol analyses); and (g) the overall cost of each regime (including the cost of office visits, endoscopy plus biopsy, rapid urease test, histology, culture and antimicrobial susceptibility testing, 13C-urea breath test, and cost of drugs). The following variables were also extracted: year of publication, format (abstract or journal article).

\section{Statistical analysis}

The primary study outcome for the meta-analysis was the eradication rate of standard triple therapy compared to culture-guided triple therapy, and the overall cost of each regime was the second outcome. Following data extraction, eradication rates from intention-to-treat (ITT) analyses and per-protocol (PP) analyses were entered into Review Manager 5.0 software programs (Cochrane Collaboration) for the performance of meta-analysis. In summarizing the data from these comparative trials, risk ratio (RR) was used as the measure of association, and summary relative RRs along with its $95 \%$ confidence interval (CI) were calculated based on a fixed-effects model using the methods of DerSimonian and Laird (38). A test of heterogeneity was also performed using the $\mathrm{I}^{2}$ statistic to establish if any clinical, methodological, or statistical variability existed among the studies used in meta-analysis. Statistical significance for the test of heterogeneity was set at 0.10 . The same method of metaanalysis was applied to the overall cost of each regime. 


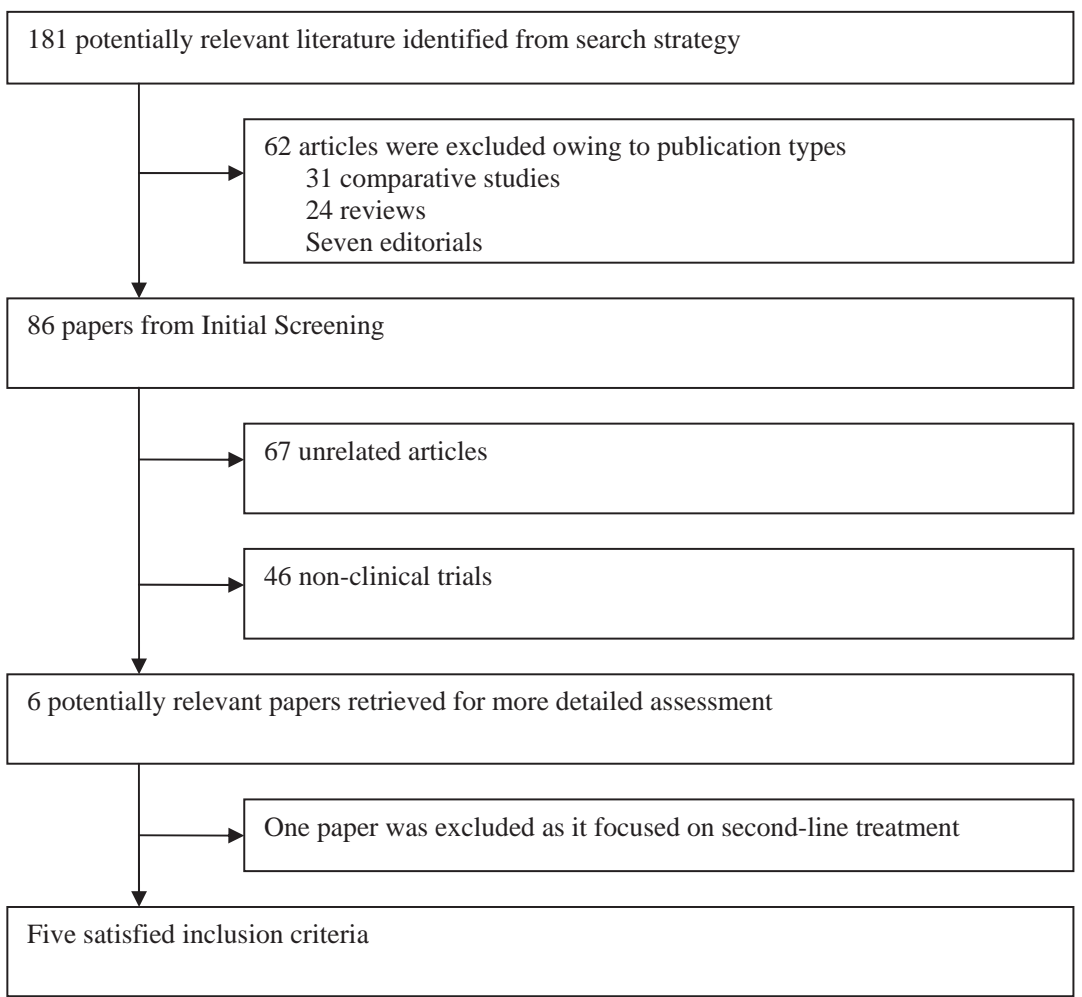

Figure 1. The flowchart of study selection.

Table 1. Quality Assessment of Included Studies (28-32)

\begin{tabular}{|c|c|c|c|c|c|c|}
\hline Study (Country) & Randomization & Allocated Concealment & Blinding & Withdrawal/lost to follow-up & Follow-up Time (w) & Dropout (n) \\
\hline Wang 2008 China & Quasi-Random & Unclear & Not described & Described & $4 \mathrm{w}$ & 9 \\
\hline $\begin{array}{l}\text { Neri } 2003 \text { Italy } \\
\text { Ramano }\end{array}$ & Unclear & Unclear & Not described & Described & $8 w$ & 10 \\
\hline $\begin{array}{l}2003 \text { Italy } \\
\text { Tarocchio }\end{array}$ & Adequate & Unclear & Not described & Described & $12 \mathrm{w}$ & 4 \\
\hline $\begin{array}{c}2000 \text { Italy } \\
\text { Ramano }\end{array}$ & Quasi-Random & Unclear & Not described & Described & $4 \mathrm{w}$ & 8 \\
\hline 2000 Italy & Unclear & Unclear & Not described & Described & $12 \mathrm{w}$ & 2 \\
\hline
\end{tabular}

w, Week; n, number

\section{Results}

The flowchart of reviews showed the detailed process of selection (Fig. 1). Of the six potential eligible articles, we excluded another one which focused on second-line treatment for H. pylori infection (39). Five RCTs ( $\mathrm{n}=701$ patients) were included at last (28-32). The quality of the included studies is shown in Table 1. The included patients did not differ in baseline characteristics (see Table 2).

The results of the five studies are summarized in Table 3. A meta-analysis of the eradication rates with ITT and PP analyses demonstrated superiority of the culture-guided triple therapy to standard triple therapy (RR, 0.84; $95 \%$ CI,0.77, 0.90; $\mathrm{p}<0.00001$ and RR, 0.83; 95\% CI,0.78, 0.89; $\mathrm{p}<0.00001$, respectively) (see Figs. 2, 3).

Only one article focused on the overall cost of cultureguided triple therapy and standard triple therapy (31). The result was approximately $\$ 5 \mathrm{U}$. S. saving per patient compared with standard triple therapy, even with the supplementary cost of culture and testing.

\section{Discussion}

Antimicrobial drug resistance can be either primary (i.e., existing before therapy) or secondary (i.e., developing as the result of failed therapy). Secondary resistance is largely responsible for the decline in eradication rates, and yet the results of comparative studies of different therapies for $H$. pylori infection that have produced very low cure rates continue to be described as equivalent to one another and as acceptable therapy (40-42). Therefore therapies for $H$. pylori infection were largely derived by using a 'hit or miss' process (43). Recently, Pilotto et al warned that the incidence of secondary $H$. pylori resistance to metronidazole and/or clarithromycin in treatment failures after 1-week proton pump inhibitor-based triple therapies was approximately $70 \%$ (44). Obviously, one of the main goals today in $H$. pylori therapy is to decrease the number of eradication failures as best as possible (45).

To solve this problem, pretherapy antibiotic sensitivity testing is one of the most promising ways, which has been suggested in high antibiotic resistance regions $(23,46)$, in 


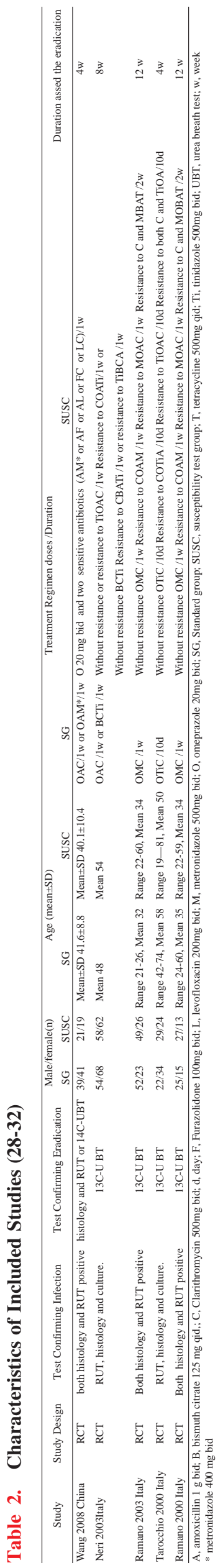

younger patients who are more likely to have been exposed to those drugs and more likely to experience treatment failure, $(47,48)$ and in children with recurrent abdominal pain or other symptoms compatible with $H$. pylori infection (49-51).

Neri et al questioned the importance of bacterial resistance to antibiotics in determining the reduction in $H$. pylori eradication efficacy (32). They stressed that many other factors influence the eradication rates of anti-H. pylori treatments, such as alcohol consumption, gender, smoking status, age, type and severity of gastritis (52), lack of gastric druginduced alkalinization (53), bacterial load (54) and the persistence of the bacterium in inaccessible sanctuaries (55). Faber et al questioned the cost-effectiveness of sensitivitybased treatment after constructing a hypothetical arithmetical model and the result was that only $5 \%$ of the patients are expected to benefit from culture-guided therapy (33). Qasim et al calculated the eradication costs from data provided by Romano et al, and pointed out that the mere 5\% eradication benefit achieved by an invasive approach may not be justifiable for the extra cost of $\$ 148 /$ patient for pretreatment susceptibility testing among young dyspeptic patients (36).

Our study shows that, standard triple therapy eradicated H. pylori infection in $75 \%$ of patients, and personalized therapy in patients affected by $H$. pylori infection significantly enhanced the eradication rate to values that were close to $90 \%$. Ideally, the regimen should be based on pretreatment drug susceptibility testing, just as in other bacterial infectious diseases (43). Only by this way clinicians can predict treatment success and the development of resistance to therapy in the community is quickly recognized, which results in rapid changes in practice to maintain excellent results.

In spite of this, the routine use of endoscopy is not feasible since it is expensive and not well-tolerated by all patients. Other less invasive and less expensive methods need to be designed for collecting specimens of $H$. pylori and for culturing it (56).

This is the first meta-analysis comparing culture-guided triple therapy versus standard triple therapy for the treatment of $H$. pylori infection. Several methodological weaknesses may limit the validity and generalizability of this metaanalysis. Initially, of the six included studies, two of them are Quasi-RCTs, none of them mentioned double blind, without which it is possible to produce high performance bias and measuring bias; none of them mentioned allocation concealment, without which might bring selective bias in these trials, because it was possible for those responsible for recruiting the participants to alter their assignment if allocation was concealed. There is also the theoretical concern for publication bias in favor of new therapy. Lastly, there are no studies involving patients from America, Africa or Japan. The studies included were conducted in Italy and China. It is well established that antibiotic resistance profiles of $H$. pylori vary among different geographical regions as pharmacogenomic difference could exist (15), which may limit the 
Table 3. Efficacy on H. Pylori Eradication (28-32)

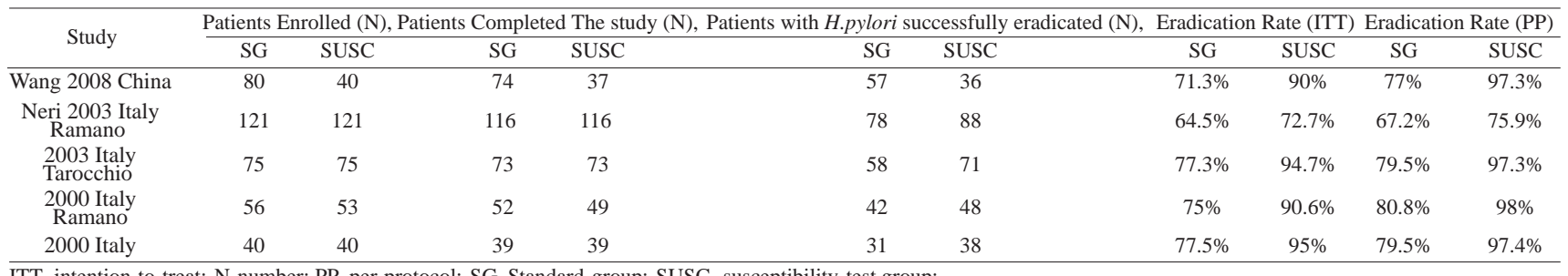

ITT, intention-to-treat; N number; PP, per-protocol; SG, Standard group; SUSC, susceptibility test group;

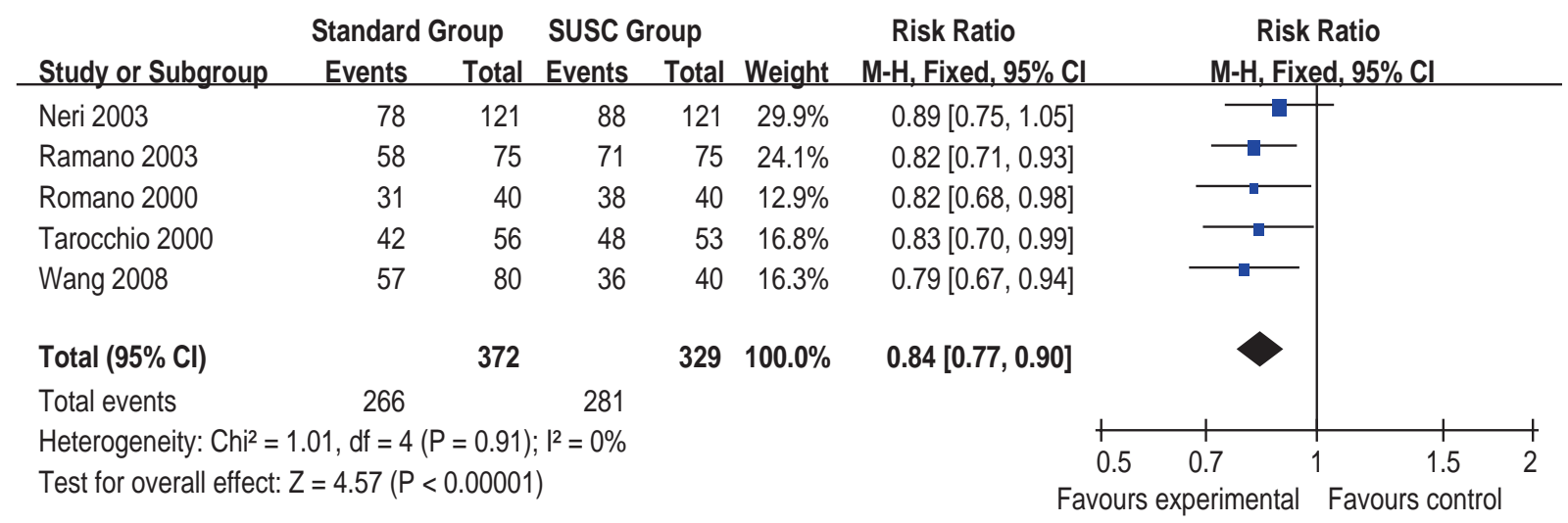

Figure 2. Efficacy on $\boldsymbol{H}$. pylori eradication-ITT.

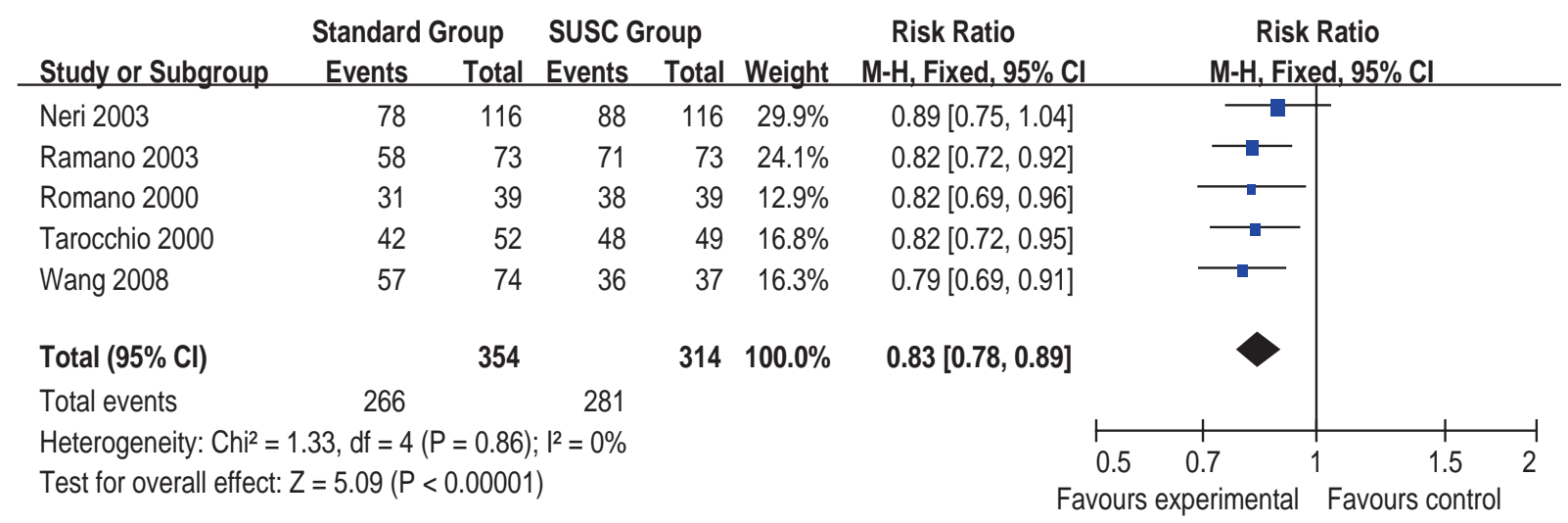

Figure 3. Efficacy on $H$. pylori eradication-PP.

generalizability of these results to Africa or other populations. Therefore, there is the need for more well-designed, double-blinded RCTs from other countries and regions.

\section{Conclusion}

In conclusion, our analysis demonstrates that cultureguided triple therapy was more effective than standard triple therapy for first-line treatment of $H$. pylori infection. Based on the only paper which focused on the overall cost, the culture-guided triple therapy was also more cost saving. Antimicrobial susceptibility testing is necessary before first-line treatment for $H$. pylori infection, and clinicians should be reminded that the antimicrobial susceptibility testing should be taken into account during the course of diagnosis and culture-guided triple therapy should be chosen for the treatment of $H$. pylori infection, particularly in high antibiotic re- sistance regions. And more well-designed, double-blind, and wide ranging geographical RCTs are still needed.

\section{Acknowledgement}

This study was founded by the Natural Science Foundation of China (No.30870364), and not funded by any pharmaceutical company.

\section{Potential Conflict of Interest}

None known. 


\section{References}

1. Proceedings of the American Digestive Health Foundation International Update Conference on Helicobacter pylori. McLean, Virginia, USA, February 13-16, 1997. Gastroenterology 113 (6 Suppl): S1-S169, 1997.

2. Peterson WL, Fendrick AM, Cave DR, Peura DA, GarabedianRuffalo SM, Laine L. Helicobacter pylori-related disease: guidelines for testing and treatment. Arch Intern Med 160: 1285-1291, 2000.

3. Hunt R, Xiao S, Megraud F, et al. World Gastroenterology Organization (WGO-OMGE) Practice Guideline: Helicobacter pylori in Developing Countries. Chin J Gastroenterol 12: 40-52, 2007.

4. Parsonnet J, Hansen S, Rodriguez L, et al. Helicobacter pylori infection and gastric lymphoma. N Engl J Med 330: 1267-1271, 1994.

5. Suerbaum S, Michetti P. Helicobacter pylori infection. N Engl J Med 347: 1175-1186, 2002.

6. Uemura N, Okamoto S, Yamamoto S, et al. Helicobacter pylori infection and the development of gastric cancer. N Engl J Med 345: 784-789, 2001.

7. Bazzoli F. Italian omeprazole triple therapy--a 1-week regimen. Scand J Gastroenterol Suppl 215: 118, 1996.

8. Lam SK, Talley NJ. Report of the 1997 Asia Pacific Consensus Conference on the management of Helicobacter pylori infection. J Gastroenterol Hepatol 13: 1-12, 1998.

9. Malfertheiner P, Megraud F, O'Morain C, et al. Current concepts in the management of Helicobacter pylori infection--the Maastricht 2-2000 Consensus Report. Aliment Pharmacol Ther 16: 167180, 2002.

10. Bazzoli F, Pozzato P. Therapy of H. pylori infection. J Physiol Pharmacol 48 Suppl 4: 39-46, 1997.

11. Pipkin GA, Williamson R, Wood JR. Review article: one-week clarithromycin triple therapy regimens for eradication of Helicobacter pylori. Aliment Pharmacol Ther 12: 823-837, 1998.

12. Graham DY. Antibiotic resistance in Helicobacter pylori: implications for therapy. Gastroenterology 115: 1272-1277, 1998.

13. Houben MH, van de Beek D, Hensen EF, Craen AJ, Rauws EA, Tytgat GN. A systematic review of Helicobacter pylori eradication therapy--the impact of antimicrobial resistance on eradication rates. Aliment Pharmacol Ther 13: 1047-1055, 1999.

14. Megraud F. Epidemiology and mechanism of antibiotic resistance in Helicobacter pylori. Gastroenterology 115: 1278-1282, 1998.

15. Megraud F. $H$ pylori antibiotic resistance: prevalence, importance, and advances in testing. Gut 53: 1374-1384, 2004.

16. Megraud F, Doermann HP. Clinical relevance of resistant strains of Helicobacter pylori: a review of current data. Gut $\mathbf{4 3}$ Suppl 1: S61-S65, 1998.

17. Ducons JA, Santolaria S, Guirao R, Ferrero M, Montoro M, Gomollon F. Impact of clarithromycin resistance on the effectiveness of a regimen for Helicobacter pylori: a prospective study of 1week lansoprazole, amoxycillin and clarithromycin in active peptic ulcer. Aliment Pharmacol Ther 13: 775-780, 1999.

18. Megraud F. Antibiotic resistance in Helicobacter pylori infection. Br Med Bull 54: 207-216, 1998.

19. Debets-Ossenkopp YJ, Herscheid AJ, Pot RG, Kuipers EJ, Kusters JG, Vandenbroucke-Grauls CM. Prevalence of Helicobacter pylori resistance to metronidazole, clarithromycin, amoxycillin, tetracycline and trovafloxacin in The Netherlands. J Antimicrob Chemother 43: 511-515, 1999.

20. Meyer JM, Silliman NP, Wang W, et al. Risk factors for Helicobacter pylori resistance in the United States: the surveillance of $H$. pylori antimicrobial resistance partnership (SHARP) study, 19931999. Ann Intern Med 136: 13-24, 2002.

21. Megraud F. Resistance of Helicobacter pylori to antibiotics. Ali- ment Pharmacol Ther 11 Suppl 1: 43-53, 1997.

22. Adamek RJ, Suerbaum S, Pfaffenbach B, Opferkuch W. Primary and acquired Helicobacter pylori resistance to clarithromycin, metronidazole, and amoxicillin--influence on treatment outcome. Am J Gastroenterol 93: 386-389, 1998.

23. Dore MP, Leandro G, Realdi G, Sepulveda AR, Graham DY. Effect of pretreatment antibiotic resistance to metronidazole and clarithromycin on outcome of Helicobacter pylori therapy: a metaanalytical approach. Dig Dis Sci 45: 68-76, 2000.

24. Pilotto A, Leandro G, Franceschi M, et al. The effect of antibiotic resistance on the outcome of three 1-week triple therapies against Helicobacter pylori. Alimentary Pharmacology and Therapeutics 13: 667-673, 1999.

25. Realdi G, Dore MP, Piana A, et al. Pretreatment antibiotic resistance in Helicobacter pylori infection: results of three randomized controlled studies. Helicobacter 4: 106-112, 1999.

26. Glupczynski Y, Megraud F, Lopez-Brea M, Andersen LP. European multicentre survey of in vitro antimicrobial resistance in Helicobacter pylori. Eur J Clin Microbiol Infect Dis 20: 820-823, 2001.

27. Ecclissato C, Marchioretto MA, Mendonca S, et al. Increased primary resistance to recommended antibiotics negatively affects Helicobacter pylori eradication. Helicobacter 7: 53-59, 2002.

28. Wang G, Zhao Q, Li S. Study of drug sensitivity test in Helicobacter pylori eradication therapy. J Clin InternMed 25: 474-477, 2008.

29. Toracchio S, Cellini L, Di Campli E, et al. Role of antimicrobial susceptibility testing on efficacy of triple therapy in Helicobacter pylori eradication. Aliment Pharmacol Ther 14: 1639-1643, 2000.

30. Romano M, Iovene MR, Montella F, Vitale LM, De Simone T, Del Vecchio Blanco C. Pretreatment antimicrobial-susceptibility testing in the eradication of $H$. pylori infection. Am J Gastroenterol 95: 3317-3318, 2000.

31. Romano M, Marmo R, Cuomo A, et al. Pretreatment antimicrobial susceptibility testing is cost saving in the eradication of Helicobacter pylori. Clin Gastroenterol Hepatol 1: 273-278, 2003.

32. Neri M, Milano A, Laterza F, et al. Role of antibiotic sensitivity testing before first-line Helicobacter pylori eradication treatments. Aliment Pharmacol Ther 18: 821-827, 2003.

33. Faber J, Bar-Meir M, Rudensky B, et al. Treatment regimens for Helicobacter pylori infection in children: is in vitro susceptibility testing helpful? J Pediatr Gastroenterol Nutr 40: 571-574, 2005.

34. Gomollon F, Sicilia B, Ducons JA, Sierra E, Revillo MJ, Ferrero M. Third line treatment for Helicobacter pylori: a prospective, culture-guided study in peptic ulcer patients. Aliment Pharmacol Ther 14: 1335-1338, 2000.

35. Breuer T, Graham DY. Costs of diagnosis and treatment of Helicobacter pylori infection: when does choosing the treatment regimen based on susceptibility testing become cost effective? Am J Gastroenterol 94: 725-729, 1999.

36. Qasim A, Sebastian S, Buckley M, O'Connor H, O'Morain C. Pretreatment antimicrobial susceptibility testing is not cost saving in the standard eradication of Helicobacter pylori. Clin Gastroenterol Hepatol. 2: 85, 2004; discussion.

37. Higgins JPT GS e. Cochrane Handbook for Systematic Reviews of Interventions. Cochrane Library. John Wiley, Chichester 2006.

38. DerSimonian R, Laird N. Meta-analysis in clinical trials. Control Clin Trials 7: 177-188, 1986.

39. Miwa H, Nagahara A, Kurosawa A, et al. Is antimicrobial susceptibility testing necessary before second-line treatment for Helicobacter pylori infection? Aliment Pharmacol Ther 17: 1545-1551, 2003.

40. Fuccio L, Minardi ME, Zagari RM, Grilli D, Magrini N, Bazzoli 
F. Meta-analysis: duration of first-line proton-pump inhibitor based triple therapy for Helicobacter pylori eradication. Ann Intern Med 147: 553-562, 2007.

41. Vakil N, Lanza F, Schwartz H, Barth J. Seven-day therapy for Helicobacter pylori in the United States. Aliment Pharmacol Ther 20: 99-107, 2004.

42. Zagari RM, Bianchi-Porro G, Fiocca R, Gasbarrini G, Roda E, Bazzoli F. Comparison of 1 and 2 weeks of omeprazole, amoxicillin and clarithromycin treatment for Helicobacter pylori eradication: the HYPER Study. Gut 56: 475-459, 2007.

43. Graham DY, Shiotani A. New concepts of resistance in the treatment of Helicobacter pylori infections. Nat Clin Pract Gastroenterol Hepatol 5: 321-331, 2008.

44. Pilotto A, Franceschi M, Rassu M, et al. Incidence of secondary Helicobacter pylori resistance to antibiotics in treatment failures after 1-week proton pump inhibitor-based triple therapies: a prospective study. Dig Liver Dis 32: 667-672, 2000.

45. de Boer WA, Borody TJ. Treatment failures and secondary resistance to antibiotics. A growing concern in Helicobacter pylori therapy. Dig Liver Dis 32: 673-675, 2000.

46. Malfertheiner P, Megraud F, O'Morain C, et al. Current concepts in the management of Helicobacter pylori infection: the Maastricht III Consensus Report. Gut 56: 772-781, 2007.

47. Gisbert JP, Luna M, Gomez B, et al. Recurrence of Helicobacter pylori infection after several eradication therapies: long-term follow-up of 1000 patients. Aliment Pharmacol Ther 23: 713-719, 2006.

48. Broutet N, Tchamgoue S, Pereira E, Lamouliatte H, Salamon R, Megraud F. Risk factors for failure of Helicobacter pylori therapy--results of an individual data analysis of 2751 patients. Aliment Pharmacol Ther 17: 99-109, 2003.

49. Drumm B, Koletzko S, Oderda G. Helicobacter pylori infection in children: a consensus statement. European Paediatric Task Force on Helicobacter pylori. J Pediatr Gastroenterol Nutr 30: 207-213, 2000.

50. Gold BD, Colletti RB, Abbott M, et al. Helicobacter pylori infection in children: recommendations for diagnosis and treatment. $\mathrm{J}$ Pediatr Gastroenterol Nutr 31: 490-497, 2000.

51. Sherman $P$, Hassall E, Hunt RH, Fallone CA, Veldhuyzen Van Zanten S, Thomson AB. Canadian Helicobacter Study Group Consensus Conference on the Approach to Helicobacter pylori Infection in Children and Adolescents. Can J Gastroenterol 13: 553$559,1999$.

52. Georgopoulos SD, Ladas SD, Karatapanis S, et al. Factors that may affect treatment outcome of triple Helicobacter pylori eradication therapy with omeprazole, amoxicillin, and clarithromycin. Dig Dis Sci 45: 63-67, 2000.

53. Furuta T, Shirai N, Takashima M, et al. Effect of genotypic differences in CYP2C19 on cure rates for Helicobacter pylori infection by triple therapy with a proton pump inhibitor, amoxicillin, and clarithromycin. Clin Pharmacol Ther 69: 158-168, 2001.

54. Perri F, Villani MR, Festa V, Quitadamo M, Andriulli A. Predictors of failure of Helicobacter pylori eradication with the standard 'Maastricht triple therapy'. Aliment Pharmacol Ther 15: 10231029, 2001.

55. Neri M, Susi D, Bovani I, Laterza F, Porreca E, Cuccurullo F. Gastric mucosal infiltration by Helicobacter pylori favours bacterial survival after treatment. Aliment Pharmacol Ther 10: 181-185, 1996.

56. Ferguson DA Jr, Jiang C, Chi DS, Laffan JJ, Li C, Thomas E. Evaluation of two string tests for obtaining gastric juice for culture, nested-PCR detection, and combined single- and doublestranded conformational polymorphism discrimination of Helicobacter pylori. Dig Dis Sci 44: 2056-2062, 1999.

(C) 2010 The Japanese Society of Internal Medicine http://www.naika.or.jp/imindex.html 